\title{
EXPLORATORY CRANIOTOMY IN COMPRESSIVE VISUAL FAILURE*
}

\author{
BY \\ F. CLIFFORD ROSE AND ALAN E. RICHARDSON \\ St. George's Hospital, and Royal Eye Medical Ophthalmology Unit, Lambeth Hospital, London
}

OF THE many causes of progressive visual failure, one of the most important is that due to compression of the optic nerve or chiasm by a surgically treatable lesion. In most instances the nature of the visual defect or the results of radiological contrast studies make the position quite clear, and dictate the appropriate management. Occasionally, however, the character of the visual defect may be unusual, and radiological studies either negative or equivocal, making diagnosis uncertain and the indication for surgical exploration a matter of judgement.

Four cases of progressive visual failure are reported here in which investigations were not conclusive in indicating the compressive lesion, but in which exploratory craniotomy revealed a tumour.

\section{Case Reports}

Case 1.-A medical practitioner aged 54 years was admitted to Atkinson Morley's Hospital on October 29, 1963, complaining of poor vision in the left eye. This had begun two and a half years previously when he noticed slight blurring of letters when he was reading; this progressed gradually over several weeks before he was convinced that it was real, but as he was a lecturer in optics he realized that the visual field showed a scotoma in the left eye, colours appearing desaturated to the left of fixation point. The blurring continued to progress and when he was seen in 1961, numerous scattered scotomata were found in the left eye, most prominent in the temporal half of the visual field; the discs were reported normal at this time. Early in 1963 the left disc was found to be paler than the right; a left carotid arteriogram was performed and was normal. His vision continued to deteriorate and in October, 1963, he could only perceive light in the left eye. On direct questioning, he admitted to having had constant vertical headaches one year previously which lasted for a few months and were worse on coughing. In the last few months he had noticed an occasional ache in the left eyeball.

On Examination.-Right visual acuity was $6 / 6$ and left visual acuity perception of light. The right visual field was full and the optic disc was normal, but the left was pale. The right pupil was normal, but the left was slightly irregular and reacted sluggishly to light directly, producing a sluggish consensual reaction in the right eye. There were no other abnormal signs on examination other than some mild bilateral perceptive deafness, the right worse than the left.

Investigations.-These, including haemoglobin estimation, white blood cell count, erythrocyte sedimentation rate, blood urea, liver function tests, and serum transaminases, were all normal. A lumbar puncture was performed and the cerebrospinal fluid was under a normal pressure, but the protein content was $75 \mathrm{mg}$. per cent. Radiographs of the chest were normal, but radiography of the skull showed a double floor of the sella turcica and a suggestion of under-cutting of the left anterior clinoid process. A lumbar air encephalogram was performed and the cerebrospinal fluid protein level at this time was $50 \mathrm{mg}$. per cent.; the lateral ventricles were moderately dilated, but there was no displacement, nor was there any unequivocal evidence of a suprasellar mass. Both the pontine and interpeduncular cisterns were larger than normal.

On November 27, 1963, left frontal craniotomy was performed, and when the arachnoid around the left optic nerve had been divided the anterior portion of a slightly reddish tumour was seen,

\footnotetext{
* Received for publication March 25, 1965.
} 
which appeared to be lateral to the optic nerve. After further dissection, it appeared that the lesion was extracerebral and appeared to be stretching the left optic nerve. When the tumour was drawn forwards the internal carotid artery was seen and this was displaced posteriorly and medially. The tumour capsule was removed piecemeal and its origin appeared to be from the lateral aspect of the sella. Microscopic section showed the tumour to be a pituitary adenoma. The patient's post-operative convalescence was uneventful and he was given a course of radiotherapy for the tumour. The visual acuity of his left eye improved from perception of light to 6/9 within six months of the operation.

Comment.-This 54-year-old lecturer in optics gave a two and a half years' history of unilateral visual failure which spread to render him virtually blind in that eye. Straight radiography of the skull and previous arteriography had been normal, as was air encephalography on his admission. The more recent straight radiographs, however, were suggestive of an intrasellar tumour and the cerebrospinal fluid protein was raised; these findings supported the need for exploratory craniotomy.

Case 2.-A woman aged 52 years was admitted (under the care of Dr. Denis Williams) on February 13,1963 . She had been well until one month before admission, when she noticed mistiness in the centre of the right field of vision. This spread to the temporal and inferior aspect of the field so that she had an area of normal vision only in the upper nasal quadrant. The left eye was not affected, but she had had occasional episodes in which she saw wavy white lines in the upper temporal quadrants of both fields, left more than right; these lasted for several minutes and occurred at intervals of several weeks during the few months prior to admission. Eight years previously right radical mastectomy had been performed for carcinoma of the breast and two and a half years before she developed diabetes insipidus which was treated successfully with pitressin injections.

On Examination.-There was a right radical mastectomy scar. Left visual acuity was $6 / 6$ and right visual acuity was limited to hand movements in the area of field defect. The right visual field showed a lower temporal field defect involving macular vision and there was a suggestion of temporal constriction of the left visual field. The right pupil was larger than the left and reacted more sluggishly to light. There were no other abnormal signs in the central nervous system.

Investigations.-The haemoglobin and white blood cell count were normal and the erythrocyte sedimentation rate was $46 \mathrm{~mm} . / 1 \mathrm{hr}$. A lumbar puncture was performed and the cerebrospinal fluid pressure and analysis were normal. Radiographs of the chest and skull were normal, as was an ultrasonogram and lumbar air encephalography. Right frontal craniotomy was performed on February 20,1963, and the right optic nerve was seen to be stretched over a tumour passing between the optic nerves and backwards under the chiasm. The tumour was solid, flat-topped, and reddishgrey and was removed piecemeal as the chiasm was prefixed; histological examination confirmed that it was a metastasis from a well-differentiated carcinoma, consistent with a primary in the breast. Post-operative convalescence was uneventful and the patient was treated with radiotherapy.

In January, 1964, she was still well on pitressin and cortisone, the visual acuity being normal on each side, but in April, 1964, she again complained of poor vision in the right eye and examination revealed a right paracentral scotoma impinging on the macula; right visual acuity was now 6/60. She was given a further course of radiotherapy and vision was restored to normal when she was last seen in July, 1964.

Comment.-In this 52-year-old woman with a one-month history of rapidly progressive deterioration of vision in the right eye radiography of the skull was normal, but the sedimentation rate was raised, and the history of carcinoma of the breast suggested that the tumour might be a metastasis. Radiography, air encephalography, and ultrasonography failed to indicate a lesion, but craniotomy revealed a breast metastasis which was removed. The rapid recovery of vision following operation and persistent good health one year after operation emphasizes that even when compressive visual failure is due to a malignant tumour, its removal is still indicated. 
Case 3.-A woman aged 48 years was admitted (under the care of Mr. Wylie McKissock) on December 28, 1963, with a history that six months previously she had noticed blurring of vision in the right eye; this was particularly marked in the central part of the field, but the visual defect had slowly progressed.

On Examination.-There was no abnormality in the general examination or in the central nervous system, except for the right eye. Left visual acuity was $6 / 5$ and the right $6 / 18$. The fundi were normal and there was no pallor of the optic discs. The left visual field was normal but on the right there was diminution of the right temporal field. The $3 / 2000$ white isopter extended into the lower nasal field, the 7/2000 red showed complete loss of the right temporal field with some encroachment of the right lower nasal field and minimal involvement of the upper nasal field.

Investigations.-The haemoglobin, white blood cell count, and erythrocyte sedimentation rate were normal, as were electro-encephalography, radiography of the skull, and right carotid arteriography. A lumbar puncture showed the cerebrospinal fluid to be under normal pressure $(120 \mathrm{~mm}$. of water) with a protein content of $10 \mathrm{mg}$. per cent. and no abnormality. Air encephalography showed a normal ventricular system with good filling of the pontine inter-peduncular and chiasmatic cisterns, but there was a suspicious filling defect present, compatible with a tumour. Right frontal craniotomy was performed on January 8, 1964, and after retraction of the right frontal lobe, an encapsulated tumour was visible on the medial aspect of the right optic nerve which was broadened and kinked upwards. Several large fragments of the tumour were removed and it was found to be large and extending under the right optic nerve and posteriorly under the chiasm. The whole of the tumour was drawn forwards, found to be lightly attached to the sella diaphragm, and removed completely. Inspection showed the sella to be intact; the only bleeding point was from a small branch from the right ophthalmic artery whence the tumour derived its blood supply. Post-operative convalescence was uneventful and the visual acuity of the right eye rapidly improved to $6 / 6$ within one week after the operation.

Comment.-This was a 48-year-old woman with a six months' history of progressive visual failure in one eye in whom all investigations, except for an equivocal air encephalogram were negative, but exploratory craniotomy revealed a meningioma which was completely removed with restoration of vision to normal.

Case 4.-A woman aged 66 years was admitted to the Royal Eye Hospital, London (under the care of Miss Doreen Birks), on October 22, 1964. She had first been seen by another ophthalmologist in 1959 with a two-year history of attacks of blurred vision, headaches, and nausea; examination at that time revealed no abnormality except a left visual acuity of $6 / 24$ and a right of $6 / 36$, which was explained by "a combination of lens changes and anxiety state".

On Examination.-Left visual acuity was 6/24, but the right had dropped to counting fingers. The visual field showed severe contraction, the right more than the left. The right pupil reacted sluggishly to direct light but briskly to consensual light and accommodation; the left pupil was normal. The left fundus was normal, but the right showed temporal pallor of the disc.

Investigations.-Haemoglobin, white blood cell count, and erythrocyte sedimentation rate were normal. Radiography of the skull showed an indefinite abnormality of the pituitary fossa, and both air encephalography and right carotid arteriography showed only minimal changes. A magnascan (isotope encephalography), performed with radioactive mercury (Spencer, 1965; Bull and Marryat, 1965), showed a well-defined circumscribed area of increased uptake in the midline above the pituitary fossa. Exploratory craniotomy was undertaken and a large suprasellar meningioma was completely removed. Following operation there was further deterioration of the patient's vision and when she was seen as an out-patient in February, 1965, the left visual acuity was limited to hand movements and the right to perception of light.

Comment.-This 66-year-old woman had a seven-year history of poor vision which was initially considered to be due to an anxiety state. Objective evidence for organic disease was a sluggish pupillary reaction on the right, where the disc was slightly pale. Special neuro-radiological techniques were unhelpful, but a magnascan using radio-active mercury indicated a suprasellar tumour; a large meningioma was removed at craniotomy, but visual acuity was worse following operation. 


\section{Discussion}

These four cases all had in common progressive visual deterioration, which was unilateral in Cases 1 and 3 and predominantly unilateral in Cases 2 and 4 . Investigations were either normal or equivocal in all four, although one investigation, differing in each case, indicated that disease was present-the raised cerebrospinal fluid protein in Case 1; the raised erythrocyte sedimentation rate in Case 2; the suspiciously abnormal air encephalogram in Case 3; and the magnascan in Case 4. It is therefore wise in any case of suspected optic nerve compression to perform all available diagnostic procedures, and when these are negative, exploratory craniotomy should be considered.

In any case of deterioration of visual acuity or progressive field loss, the first step is to exclude ocular causes. Glaucoma is usually easily diagnosed by the type of headache, "halo" vision, cupped disc, arcuate scotoma, and raised intra-ocular pressure on tonometry. Vascular causes have an acute onset and are not progressive, whereas retinal lesions will be recognized on funduscopy. Optic and retrobulbar neuritis can be distinguished by the rapid onset, pain on eye movement, and globe tenderness, and the optic nerve head may be oedematous; the scotoma is usually central and recovery of vision is the rule within a few weeks (Meadows, 1949; Rose, 1964, 1965). Furthermore, the age range of 15 to 45 years is younger than that usually found in cases of meningioma, metastases, aneurysm, or chromophobe adenoma - the commonest causes of compression of the optic nerve or chiasm.

Case 1 presented initially with a para-central scotoma which later spread to the periphery, and this "breaking-through" is typical of optic nerve compression (Meadows, 1949), presumably because the central macular fibres are more sensitive to pressure, because of their different size or their vascularity. The tumour in Case 1 was a chromophobe adenoma which characteristically presents with bitemporal hemianopia due to chiasmal compression. Patients with pituitary tumours may misleadingly complain of poor vision in one eye, but careful perimetry usually reveals an upper temporal field defect, if only to colour, on the opposite side. When, rarely, the field defect is confined to one eye, it is usually a temporal hemianopia and a unilateral central scotoma is exceptional; in a series of 256 cases of pituitary tumour seen at Atkinson Morley's Hospital, only one presented in this manner (Elkington, 1964). The loss of vision in one eye only has been explained by Walsh (1957) on the basis of the tumour being pre-chiasmal, but he, too, had seen only one case presenting with a unilateral central scotoma.

The marked improvement in vision following removal of the tumour in the first three cases was gratifying; in Case 1, in spite of a history of over two years and optic atrophy, the visual acuity increased from light perception to $6 / 9$, the improvement taking up to six months. In Case 2, in spite of the compressive lesion being malignant, the visual acuity improved from hand movements to normal. In Case 3 the improvement in visual acuity occurred within a few days of operation. In Case 4 the visual acuity deteriorated further following operation, and this not infrequently occurs when a tumour has been present for many years. We do not ascribe it to the more difficult removal of a larger growth, for the optic pathways are carefully avoided during operation; the more likely explanation is that following removal of the neoplasm there are petechial haemorrhages into the optic nerves which have 
been rendered ischaemic from pressure. Vision may thus improve considerably after operation even though the acuity is light perception only and even in the presence of optic pallor; improvement can occur within a few days, but may continue up to several months, but in order to obtain a good result, surgical removal must be made early. Case 2 illustrates the need for a more active approach to intracranial metastases, particularly when these arise from breast cancer; about 6 per cent. of intracranial secondaries are from a primary in the breast, but of those patients with cerebral metastases who survive more than a year, over 25 per cent. are those with disseminated mammary carcinoma, indicating their better prognosis (Richards and McKissock, 1963).

The commonest cause of optic nerve compression is meningioma. Meadows (1949) reported a series of 11 cases, 7 of which were due to basal meningioma, 3 to aneurysms or vascular anomalies, and 1 to glioma; 4 of these cases had normal, and 1 doubtfully abnormal, investigations. The difficulty of diagnosing suprasellar tumours is that contrast radiography may be negative (Grant and Hedges, 1956) and since these tumours are benign, exploratory craniotomy must be considered. In any patients where repeated examinations of visual acuity and fields indicate a progressive lesion, full investigations, including examination of the blood and cerebrospinal fluid, radiography of the skull with coned views of the sella turcica, arteriography, air encephalography, and magnascan studies should be performed. Even when these are negative, rather than risk leaving a removable tumour which will cause blindness, it is wiser to perform exploratory craniotomy, particularly since this operation no longer possesses the hazards it once had (Jane and McKissock, 1962).

\section{Summary}

Four cases of progressive, mainly unilateral, visual failure are reported; in spite of equivocal results of investigation, exploratory craniotomy was undertaken and a tumour found in each case. Following removal of the tumour, marked improvement occurred in vision in three cases, one of which was a metastasis from a carcinoma of the breast. A more active attitude to intracranial secondaries from the breast is advocated. In one case of suprasellar meningioma, where there was a history of over seven years, vision failed to improve following operation.

The difficulties in diagnosis and management are discussed, and the importance of periodic visual field examination and early investigation, including magnascan, is emphasized. In any suspected case of compressive visual failure, even when investigations are negative, exploratory craniotomy is indicated.

It is a pleasure to thank Dr. Denis Williams, Mr. Wylie McKissock, and Miss Doreen Birks for their permission to publish Cases 2, 3, and 4 respectively.

\section{REFERENCES}

Bull, J. W. D., and Marryat, J. (1965). Brit. med. J., 1, 474.

Elkington, S. C. (1964). Personal communication.

Grant, F. C., and HedGes, T. R. (1956). A.M.A. Arch. Ophthal., 56, 163.

JANE, J. A., and McKissocK, W. (1962). Brit. med. J., 2, 5.

Meadows, S. P. (1949). Proc. roy. Soc. Med., 42, 1017.

Richards, P., and McKissock, W. (1963). Brit. med. J., 1, 15.

Rose, F. C. (1964). Postgrad. med. J., 40, 692.

(1965). Proc. roy. Soc. med., 58, 537.

SPENCER, R. (1965). Brit. J. Radiol., 38, 1.

WALSH, F. B. (1957). “Clinical Neuro-Ophthalmology", 2nd ed. Williams and Wilkins, Baltimore. 